\title{
Scaling Laws of Collective Ride-Sharing Dynamics
}

\author{
Nora Molkenthin $\odot,{ }^{1,2,3}$ Malte Schröder $\odot,{ }^{1}$ and Marc Timme ${ }^{1,2,4}$ \\ ${ }^{1}$ Chair for Network Dynamics, Institute for Theoretical Physics and Center for Advancing Electronics Dresden (cfaed), \\ Technical University of Dresden, 01069 Dresden, Germany \\ ${ }^{2}$ Network Dynamics, Max Planck Institute for Dynamics and Self-Organization (MPIDS), 37077 Göttingen, Germany \\ ${ }^{3}$ Potsdam Institute for Climate Impact Research, P.O. Box 601203, Potsdam, 14412, Germany \\ ${ }^{4}$ Max Planck Institute for the Physics of Complex Systems (MPIPKS), 01069 Dresden, Germany
}

(Received 8 April 2020; revised 13 November 2020; accepted 16 November 2020; published 10 December 2020)

\begin{abstract}
Ride-sharing services may substantially contribute to future sustainable mobility. Their collective dynamics intricately depend on the topology of the underlying street network, the spatiotemporal demand distribution, and the dispatching algorithm. The efficiency of ride-sharing fleets is thus hard to quantify and compare in a unified way. Here, we derive an efficiency observable from the collective nonlinear dynamics and show that it exhibits a universal scaling law. For any given dispatcher, we find a common scaling that yields data collapse across qualitatively different topologies of model networks and empirical street networks from cities, islands, and rural areas. A mean-field analysis confirms this view and reveals a single scaling parameter that jointly captures the influence of network topology and demand distribution. These results further our conceptual understanding of the collective dynamics of ride-sharing fleets and support the evaluation of ride-sharing services and their transfer to previously unserviced regions or unprecedented demand patterns.
\end{abstract}

DOI: 10.1103/PhysRevLett.125.248302

Traditional forms of human mobility from pedestrian to private car traffic and air travel exhibit a range of collective dynamical phenomena including freezing by heating, congestion, traffic flow oscillations, and perturbation spreading [1-7]. Nonlinear dynamics and statistical physics have substantially contributed to identifying many of these phenomena and to providing a better understanding of the mechanisms underlying them as well as their relations to other collective dynamics [3-12].

However, mobility is currently becoming more and more digitized such that the mobile agents-humans and vehicles-interact in new, increasingly complex ways. Ride-sharing platforms [13] constitute paradigmatic examples of such networked mobility services (Fig. 1). Each traveler requests a ride from a desired origin to a desired destination at a certain time. The service provider assigns the request to one out of a large fleet of ride-sharing vehicles, such as minibuses. The routes of these vehicles are dynamically updated to simultaneously satisfy the spatiotemporal constraints of all previously assigned passengers, the vehicles themselves, and the new request. Such on-demand assignment of passengers to vehicles and

Published by the American Physical Society under the terms of the Creative Commons Attribution 4.0 International license. Further distribution of this work must maintain attribution to the author(s) and the published article's title, journal citation, and DOI. Open access publication funded by the Max Planck Society. the simultaneous multivehicle routing [14-16] drastically increases the complexity of ride-sharing platforms beyond those of traditional line-based, fixed-schedule public transport or simple taxi services. Recent studies [17-21] have characterized the economic feasibility and the theoretical potential of optimal ride-sharing services based on the spatial distributions of simultaneous requests. Yet, how efficient the dynamics of ride-sharing fleets may be, is not well understood. Estimating service performance across
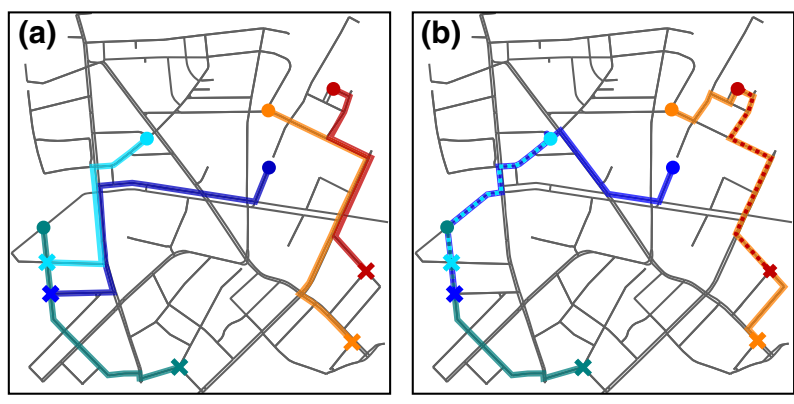

FIG. 1. Ride sharing combines similar trips to fewer vehicles. (a) Private car traffic and traditional ride-hailing services (e.g., taxis) serve every request individually (one color for each request, start and end point marked by disks and crosses, respectively). (b) Ride-sharing services reduce the total distance driven by combining similar requests. In the illustrative sketch, five requests are served by two vehicles, one serving three requests (blue), one serving two (red), exploiting substantial overlap of the respective routes. 
regions, various street network topologies and demand conditions requires an objective and transferrable measure of ride-sharing efficiency that makes services comparable across different settings.

Here, we introduce a quantitative measure of efficiency of on-demand ride-sharing fleets based on their collective nonlinear dynamics and reveal its universal scaling across network topologies. Combining direct numerical simulations and a mean-field analysis, we estimate the functional form of a universal scaling function asymptotically for large fleet sizes. The analysis uncovers a single scaling parameter that simultaneously captures the influence of the demand pattern and the topology of the street network.

The collective dynamics of a ride-sharing service crucially depends on three factors: (i) the topology of the street network, (ii) the demand distribution, i.e., the distribution of origin and destination of all requests in space and time, and (iii) the dispatcher algorithm that plans the routes of all $B$ ride-sharing vehicles (buses), dynamically updating these routes to serve all incoming requests. Here, we focus on the impact of the topology of the street network on the efficiency of ride sharing, evaluating ride-sharing dynamics on various empirical and model street networks. The demand distribution naturally enters as it modifies the importance of specific nodes and edges of the network and thereby the effective topology created by the vehicles' driving patterns.

To compare the collective dynamics of the ride-sharing fleet across topologies, demand patterns, request rates $\lambda$, fleet sizes $B$, and characteristic bus velocities $v$, we introduce a dimensionless parameter

$$
x=\frac{\langle l\rangle}{v B} \lambda,
$$

where $\langle l\rangle$ is the average distance of requested trips on a given topology. This effective system load $x$ describes the ratio of requested trip distance $\langle l\rangle \lambda$ per unit time and the total bus driving distance $v B$ per unit time. A longer average trip length $\langle l\rangle$ implies that buses are busy with individual requests longer and the effective load is higher. Increasing the driving velocity $v$ or the number $B$ of buses reduces the load per bus. If $x<1$, all requests can theoretically be served one by one (e.g., by taxis with one passenger seat), if $x>1$ ride sharing becomes necessary as buses cannot cover the requested distance with trips serving passengers individually.

To isolate the impact of the network topologies, we first consider the simplest setting: we take requests to be generated by a Poisson process of rate $\lambda$ and the origins and destinations of all trips to be independently and uniformly distributed among the nodes of an undirected street network with buses that do not reach their passenger capacity. We further consider a basic dispatcher that minimizes the arrival time of a passenger at their destination without delaying previously assigned passenger trips or reshuffling passengers among buses. All results presented in this work stay qualitatively the same under substantially more general conditions, including correlated and asymmetric demand distributions, directed street networks, and different dispatchers (see Supplemental Material [22]).

What are suitable observables to quantify ride-sharing efficiency? Instead of focusing on specific resources, such as total fuel consumption, time of operation, or monetary cost, we here evaluate efficiency based on the intrinsic fleet dynamics. At any time, each bus of a ride-sharing service is scheduled to serve a number $C$ of passengers, including passengers already on the bus as well as passengers scheduled to be picked up in the future. As the load on the system increases, more passengers per bus are scheduled and served. Figure 2 illustrates the scaling of the average number of scheduled passengers $\langle C\rangle$ for various model networks. If the number of scheduled passengers exactly reflects the load on the system, i.e., per bus the fleet serves $\langle C\rangle=x$ passengers at any given time, the system is operating at ideal, i.e., maximal theoretically possible efficiency. Consider, for example, the onset of ride sharing,
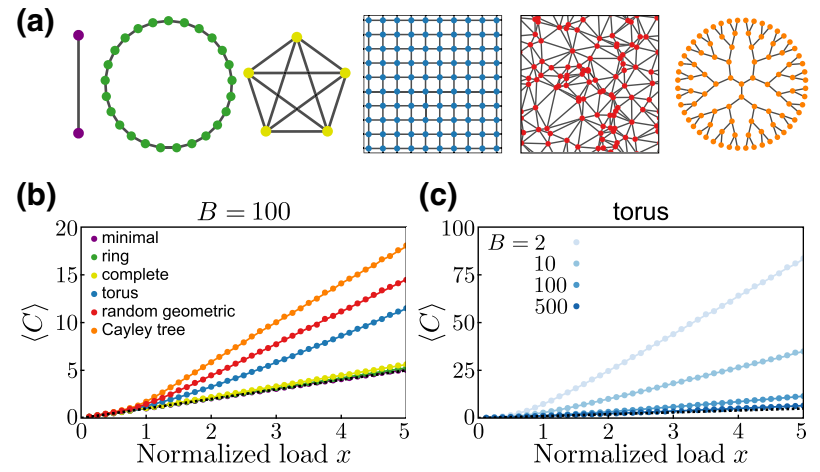

FIG. 2. Scaling of the number of scheduled passengers measures ride-sharing efficiency. (a) Model networks with qualitatively different topologies: a minimal graph $(N=2$, blue), a cycle graph (ring, $N=25$, green), a complete graph ( $N=5$, yellow), a square lattice with periodic boundaries (torus, $N=100$, blue), a random geometric network $(N=100$, red), and a Cayley tree with degree $3(N=94$, orange). (b) The average number of scheduled passengers $\langle C\rangle$ grows linearly with the normalized request rate $x$ in the ride-sharing regime $(x>1)$, shown for the different model networks each with $B=100$ buses. Ride sharing is easier in networks with few distinct shortest paths (e.g., ring, green) and $\langle C\rangle$ is closer to the optimal service scaling $\langle C\rangle=x$ (black dashed line). The colored lines indicate the expected number of passengers, confirming the mean-field prediction from the observed waiting and driving times, Eq. (6). (c) The scaling of $\langle C\rangle$ converges to the optimal scaling as the number of buses is increased, shown here for the torus network [blue in panels (a) and (b)]. At constant normalized load $x$ the number of requests increases proportionally to the number of buses, thus also increasing the number of similar trips that can be shared efficiently. 
$x=1$ : in the limit of perfectly efficient service, the ride-sharing buses on average have exactly $\langle C\rangle=1$ passenger scheduled at each time. In contrast, if the buses serve individual requests less efficiently at a lower rate, they must have $\langle C\rangle>1$ passengers scheduled. The deviation from the ideal scaling $\langle C\rangle=x$ naturally measures the efficiency

$$
E=\lim _{x \rightarrow \infty}\left(\frac{\langle C\rangle}{x}\right)^{-1}
$$

in terms of the intrinsic dynamics of the ride-sharing system. Instead of measuring the total efficiency, measuring the differential response to changes in load $x$ defines the susceptibility

$$
\chi(x)=\left(\frac{d\langle C\rangle}{d x}\right)^{-1},
$$

of the networked system [23] describing the efficiency with which the system handles additional requests. In the limit of high load, the susceptibility becomes identical to the efficiency,

$$
E=\lim _{x \rightarrow \infty} \chi(x)
$$

due to the linear scaling of $\langle C\rangle$ for large $x$ [compare Figs. 2(b) and 2(c)].

Analyzing the efficiency across a broad variety of street network topologies, ranging from one- and twodimensional lattices to random geometric graphs and from star and other tree structures to all-to-all coupled networks, we find quantitatively different dependencies on the number of buses $B$ [Fig. 3(a)].

Yet, the qualitative similarity of the efficiency curves suggests an overarching scaling feature that holds across topologies. Indeed, rescaling the number of buses by a factor $B_{1 / 2}$, such that $E\left(B_{1 / 2}\right)=1 / 2$, yields a universal scaling

$$
E=f\left(\frac{B}{B_{1 / 2}}\right)
$$

of the efficiency with the number of ride-sharing buses across topologies. As a consequence, for large $B$ the efficiency curves collapse onto a single scaling function $f(\cdot)$ [Fig. 3(b)]. We note that this collapse not only occurs in the limit $B \rightarrow \infty$ where $E=1$, but equally holds for smaller $B$ until the efficiency is as small as $E(B)=10^{-1}$ or below. We find the same original diversity [Fig. 3(a) inset] and universality after rescaling [Fig. 3(b) inset] for empirical street networks of cities of different sizes and densities, rural areas, and islands served with a different dispatcher, see Supplemental Material [22] for more details. The emergence of universality is insensitive against varying system details and holds across a range of request distributions with uncorrelated and correlated as well as symmetric and asymmetric origin-destination pairs (see
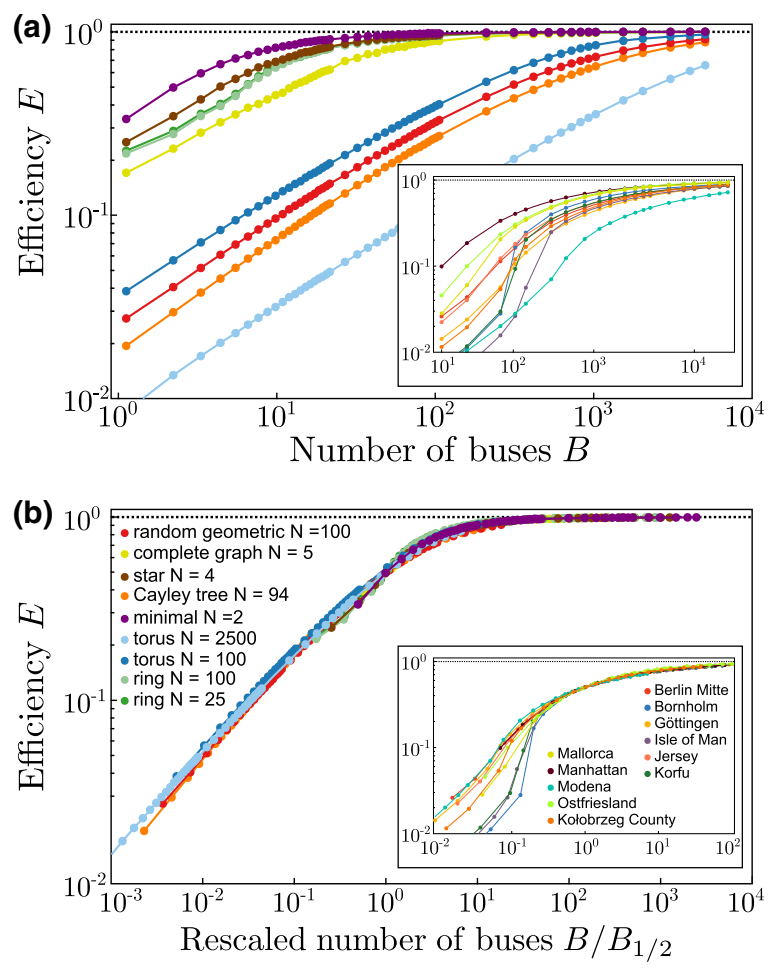

FIG. 3. Topological universality of ride-sharing efficiency. (a) The ride-sharing efficiency $E$ [Eq. (2), evaluated at $x=7.5]$ measured by the deviation from the optimal scaling $\langle C\rangle=x$ of the number of scheduled passengers in qualitatively different model networks (compare Fig. 2). The quantitative value of the efficiency varies strongly across the different topologies while the qualitative behavior is similar. In smaller networks with overlapping shortest paths the maximum efficiency $E=1$, denoting optimal service, is easier to reach. (b) The ride-sharing efficiency collapses to a universal efficiency function $f\left(B / B_{1 / 2}\right)$ [Eq. (5)] across all model networks. (Insets) We find the same qualitative behavior and topological universality across qualitatively different empirical street networks (cities, islands, rural areas): The ride-sharing efficiency collapses to a universal scaling function $f\left(B / B_{1 / 2}\right)$ for large $B$. Here, the ride-sharing efficiency $E$ was evaluated at lower $x=2.5$ and with a dispatcher that allows some delay of already scheduled requests to enable short detours in the heterogeneous street networks. We remark that the scaling functions for the model networks (main panels) and the empirical street networks, although they have similar shape, are slightly different because of two different dispatchers serving the requests, see Supplemental Material [22] for more details.

Supplemental Material [22]). Thus, this topological universality captures the influence of the topology of the street network and demand distribution in a single scaling parameter $B_{1 / 2}$ explaining most of the variability of the efficiency. Moreover, the scaling function $f(\cdot)$ changes across dispatchers, such that the rescaled efficiency function offers an objective and transferrable measure to compare the efficiency of ride-sharing dispatchers.

How does the scaling function $f(\cdot)$ relate to the observables of the ride-sharing dynamics? The number of 
scheduled passengers $\langle C\rangle$, averaged in a stationary operating state, is directly related to the average waiting time $\left\langle\Delta t_{w}\right\rangle$ until pickup and the average driving time $\left\langle\Delta t_{d}\right\rangle$ between pickup and dropoff of an individual passenger. During the average service time of a passenger between request and dropoff at the destination, $\left\langle\Delta t_{s}\right\rangle=\left\langle\Delta t_{w}\right\rangle+$ $\left\langle\Delta t_{d}\right\rangle$, the bus is assigned new requests at an average rate $\lambda / B$, thus on average scheduling a total of $\lambda\left\langle\Delta t_{s}\right\rangle / B$ new requests. On average, at the time that passenger leaves the bus, only those new requests are still to be served while the older ones have been delivered before. The average number of scheduled passengers $\langle C\rangle$ is therefore equal to the average number of requests scheduled during the service time,

$$
\langle C\rangle=\left\langle\Delta t_{s}\right\rangle \frac{\lambda}{B}=\frac{x}{\tau}\left(\left\langle\Delta t_{d}\right\rangle+\left\langle\Delta t_{w}\right\rangle\right) .
$$

Here, we denote the natural timescale in the system as $\tau=\langle l\rangle / v$, describing the average direct travel time that would arise by an instantly available taxi ride. Similarly, we can estimate the average occupancy related to the driving time as $\langle O\rangle=(x / \tau)\left\langle\Delta t_{d}\right\rangle$.

We derive the scaling of the efficiency curve from that of $\langle C\rangle$ asymptotically for large fleet size $B \rightarrow \infty$ [compare Fig. 2(c)] and for large $x \gg 1$, i.e., close to the perfect service limit as in the definition of ride-sharing efficiency, Eq. (2). For suitable asymptotically efficient ride-sharing dispatchers, the delay due to detours disappears as $B \rightarrow \infty$, such that

$$
\left\langle\Delta t_{d}\right\rangle \sim \tau \propto B^{0}
$$

to leading order in $B$.

For ideal service efficiency, the waiting time is determined by the number of buses going directly from the origin to the destination of a request. When there are sufficiently many buses in the network, multiple buses drive along each shortest path in the network. Consequently, the waiting time decays to zero inversely proportional to the number of buses, as $B^{-1}$ for large $B$. A proportionality factor $\gamma$ reflects the characteristic number of buses at which the average waiting time matches the system-intrinsic timescale $\tau$. We thus obtain

$$
\left\langle\Delta t_{w}\right\rangle \sim \gamma \tau B^{-1}
$$

asymptotically as $B \rightarrow \infty$. Substituting Eqs. (8) and (7) into Eq. (6) yields

$$
\langle C\rangle \sim \frac{x}{\tau}\left(\tau+\gamma \tau B^{-1}\right)=x\left(1+\frac{\gamma}{B}\right)
$$

and therefore

$$
E \sim f(z)=\frac{1}{1+z^{-1}}
$$

as $z \rightarrow \infty$. We directly identify $z=B / \gamma$ and thus $\gamma=B_{1 / 2}$ as the number of buses required to reach half efficiency (comparing well to simulation results, see Supplemental Material [22], Fig. S2). We remark that the above derivation of Eq. (10) includes the effect of the request distribution on the scaling factor by calculating the characteristic trip length $\langle l\rangle$ as a weighted average with respect to the request distribution.

The scaling factor $B_{1 / 2}$ reflects the difficulty of ride sharing for the given network topology and given request distribution. In networks with many overlapping shortest paths between different pairs of nodes (e.g., the cycle graph), rides can be shared easily without additional detours and $B_{1 / 2}$ is small. In networks with many distinct, nonoverlapping shortest paths (e.g., trees), sharing rides more likely requires detours, making it less efficient. Consequently, $B_{1 / 2}$ is large for these networks.

The above derivation together with additional numerical results (see Supplemental Material [22]) demonstrate the degree of robustness of the observed scaling law. The topological universality extends to nonuniform demand distributions via its dependence on the average trip length $\langle l\rangle$ and a slightly modified scaling factor $B_{1 / 2}$, reflecting the modified distribution of buses on the network. Similarly, different dispatcher algorithms or additional constraints such as a limited vehicle capacity do not qualitatively change the scaling law, provided the system is operating in the high efficiency regime where the asymptotic scaling in the above derivation holds [compare Fig. 3(b), see also Supplemental Material [22] ].

Yet, by its very nature, the universality across topologies cannot hold across arbitrary services and conditions. For instance, if the ride-sharing fleet itself generates the majority of the traffic in a city, congestion and thereby the characteristic driving velocity $v$ will explicitly depend on the request rate $\lambda$ and the number of vehicles $B$. Moreover, our derivation of the scaling assumes an asymptotically constant driving time and a waiting time scaling as $B^{-1}$ for large $B$. This states a simple dimensional scaling expected for a range of suitable dispatchers, including optimization algorithms currently employed in ride-sharing services. However, this scaling may be different in systems that are operating close to their capacity limit or with strongly heterogeneous parameters. Understanding how, why, and under which conditions the topological universality breaks down may provide insights toward enabling the design of more robust or optimized ride-sharing systems capable of operating efficiently across these different settings.

Previous work by Tachet et al. [19] found universal scaling of the potential shareability of pairs of empirical ride requests in different cities with increasing request rate 
without explicit reference to positions or dispatching of vehicles. In contrast, we observe universal scaling of ridesharing efficiency as a function of the fleet size in a dynamic model with direct simulation of vehicle routes. The existence of universal scaling in both cases may suggest a more fundamental universality across shared mobility processes on networks, for example mediated by the overlap of (shortest) paths that enable sharing rides without detours. Understanding this deeper connection may help to further improve our theoretical insights into the complex dynamics of ride sharing.

The system-intrinsic efficiency measure as well as its topological universality presented here may provide valuable insights into the nonlinear collective dynamics of ride-sharing fleets on topologically distinct street networks and offer a complementary approach to conventional efficiency measures. The universality supports the consistent evaluation of ride-sharing efficiency and feasibility under a broad variety of conditions across cities and rural areas of different sizes, densities, and with qualitatively different street networks and demand distributions [17,19]. This may help to identify network and demand structures as well as service conditions that promote efficient ride sharing as well as enable the topological optimization of stop locations or the prediction of required fleet sizes in previously unserviced areas. Moreover, variations of the scaling across dispatcher algorithms may help compare dispatchers for given conditions. The results may thus not only enable improved planning of ride-sharing fleets under untested conditions, but also the use of data-driven automated methods to select suitable dispatcher algorithms and service parameters by making data from different settings comparable, cf. [24]. Overall, the results underline the potential of analyses of the collective dynamics of modern, networked forms of mobility.

We thank Debsankha Manik, Jan Nagler, Nils Beyer, Stephan Herminghaus, Jani-Pekka Jokinen, and Verena Krall for helpful comments and fruitful discussions. We thank Robin Zech for help with additional simulations. This research was supported by the German Research Foundation (Deutsche Forschungsgemeinschaft, DFG) through the Center for Advancing Electronics Dresden (cfaed) and the European Fund for Regional Development (ERDF/EFRE) through the state of Lower Saxony, and the Max Planck Society.

[1] D. Helbing, I. J. Farkas, and T. Vicsek, Freezing by Heating in a Driven Mesoscopic System, Phys. Rev. Lett. 84, 1240 (2000).

[2] I. Karamouzas, B. Skinner, and S. J. Guy, Universal Power Law Governing Pedestrian Interactions, Phys. Rev. Lett. 113, 238701 (2014).
[3] A. Loder, L. Ambühl, M. Menendez, and K. W. Axhausen, Understanding traffic capacity of urban networks, Sci. Rep. 9, 16283 (2019).

[4] D. Li, B. Fu, Y. Wang, G. Lu, Y. Berezin, H. E. Stanley, and S. Havlin, Percolation transition in dynamical traffic network with evolving critical bottlenecks, Proc. Natl. Acad. Sci. U.S.A. 112, 669 (2015).

[5] G. Zeng, D. Li, S. Guo, L. Gao, Z. Gao, H. E. Stanley, and S. Havlin, Switch between critical percolation modes in city traffic dynamics, Proc. Natl. Acad. Sci. U.S.A. 116, 23 (2019).

[6] A. Gautreau, A. Barrat, and M. Barthélemy, Arrival time statistics in global disease spread, J. Stat. Mech. (2007) L09001.

[7] D. Brockmann and D. Helbing, The hidden geometry of complex, network-driven contagion phenomena, Science 342, 1337 (2013).

[8] D. Chowdhury, L. Santen, and A. Schadschneider, Statistical physics of vehicular traffic and some related systems, Phys. Rep. 329, 199 (2000).

[9] D. Brockmann, L. Hufnagel, and T. Geisel, The scaling laws of human travel, Nature (London) 439, 462 (2006).

[10] M. Saberi, H. S. Mahmassani, D. Brockmann, and A. Hosseini, A complex network perspective for characterizing urban travel demand patterns: Graph theoretical analysis of large-scale origin-destination demand networks, Transportation 44, 1383 (2017).

[11] S. M. Krause, L. Habel, T. Guhr, and M. Schreckenberg, The importance of antipersistence for traffic jams, Europhys. Lett. 118, 38005 (2017).

[12] W. Staffeldt and A. K. Hartmann, Rare-event properties of the Nagel-Schreckenberg model, Phys. Rev. E 100, 062301 (2019).

[13] M. Furuhata, M. Dessouky, F. Ordóñez, M.-E. Brunet, X. Wang, and S. Koenig, Ridesharing: The state-of-the-art and future directions, Transp. Res. B 57, 28 (2013).

[14] A. Sorge, Towards a statistical physics of collective mobility and demand-driven transport, Ph.D. thesis, Georg-AugustUniversität Göttingen, 2017.

[15] A. Sorge, D. Manik, S. Herminghaus, and M. Timme, Towards a unifying framework for demand-driven directed transport (D3T), in Proceedings of the 2015 Winter Simulation Conference (IEEE Press, New York, 2015), pp. 2800-2811.

[16] J. Alonso-Mora, S. Samaranayake, A. Wallar, E. Frazzoli, and D. Rus, On-demand high-capacity ride-sharing via dynamic trip-vehicle assignment, Proc. Natl. Acad. Sci. U.S.A. 114, 462 (2017).

[17] P. Santi, G. Resta, M. Szell, S. Sobolevsky, S. H. Strogatz, and C. Ratti, Quantifying the benefits of vehicle pooling with shareability networks, Proc. Natl. Acad. Sci. U.S.A. 111, 13290 (2014).

[18] J.-P. Jokinen, On the welfare optimal policies in demand responsive transportation and shared taxi services, J. Transp. Econ. Policy 50, 39 (2016), https://www.ingentaconnect .com/content/lse/jtep/2016/00000050/00000001/art00004.

[19] R. Tachet, O. Sagarra, P. Santi, G. Resta, M. Szell, S. H. Strogatz, and C. Ratti, Scaling law of urban ride sharing, Sci. Rep. 7, 42868 (2017). 
[20] M. M. Vazifeh, P. Santi, G. Resta, S. H. Strogatz, and C. Ratti, Addressing the minimum fleet problem in on-demand urban mobility, Nature (London) 557, 534 (2018).

[21] S. Herminghaus, Mean field theory of demand responsive ride pooling systems, Transp. Res. A 119, 15 (2019).

[22] See Supplemental Material at http://link.aps.org/ supplemental/10.1103/PhysRevLett.125.248302 for additional details on analytics and data.
[23] D. Manik, M. Rohden, H. Ronellenfitsch, X. Zhang, S. Hallerberg, D. Witthaut, and M. Timme, Network susceptibilities: Theory and applications, Phys. Rev. E 95, 012319 (2017).

[24] A. P. Riascos and J. L. Mateos, Networks and long-range mobility in cities: A study of more than one billion taxi trips in New York City, Sci. Rep. 10, 4022 (2020). 